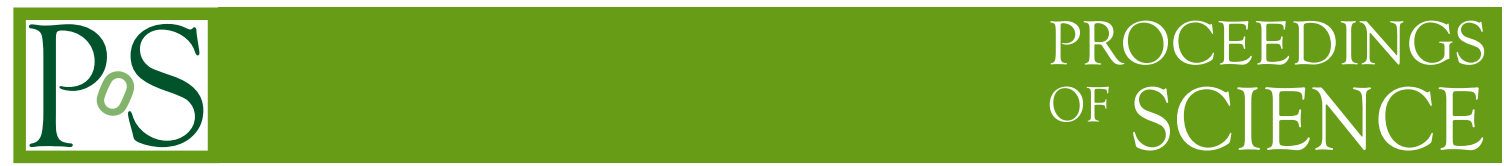

\title{
Recent results using semileptonic decays with $\mathrm{LHCb}$
}

\section{Antonio Romero Vidal* $\dagger$}

Instituto Galego de Física de Altas Enerxías (IGFAE), Universidade de Santiago de Compostela, Spain

E-mail: antonio.romero@usc.es

This document summarizes the measurements performed at the LHCb experiment using semileptonic $b$-hadron decays. Measurements of the CKM factor $\left|V_{u b}\right|$, lepton flavour universality tests by measuring the ratios $R\left(D^{*}\right)$ and $R(J / \psi)$, a search for the decay $B^{+} \rightarrow \mu^{+} \mu^{-} \mu^{+} v_{\mu}$ and the measurement of charmed-baryon lifetimes using semileptonic decays are presented.

7th Annual Conference on Large Hadron Collider Physics - LHCP2019

20-25 May, 2019

Puebla, Mexico

* Speaker.

${ }^{\dagger}$ on behalf of the LHCb collaboration. 


\section{Introduction}

The study of semileptonic $b \rightarrow c \ell^{-} \bar{v}_{\ell}$ and $b \rightarrow u \ell^{-} \bar{v}_{\ell}$ transitions, where $\ell$ represents a charged lepton, are of great importance for testing the Standard Model (SM) of particle physics. The study of these transitions allows to precisely determine the CKM parameters $\left|V_{c b}\right|$ and $\left|V_{u b}\right|$, but also to perform tests of lepton flavour universality (LFU) comparing semileptonic $b$-hadron decays into the different families of charged leptons. In addition, due to the large branching fractions of $b \rightarrow c \ell^{-} \bar{v}_{\ell}$ transitions, they have also been used to measure the lifetimes of charmed baryons. Section 2 shows the measurement of the lifetimes of the $\Xi_{c}^{+}, \Lambda_{c}^{+}, \Xi_{c}^{0}$ and $\Omega_{c}^{0}$ baryons using semileptonic $b$-hadron decays. The measurement of $\left|V_{u b}\right|$ using $\Lambda_{b}^{0} \rightarrow p \mu^{-} \bar{v}_{\mu}$ decays is presented in section 3. Section 4 includes a search for the $B^{+} \rightarrow \mu^{+} \mu^{-} \mu^{+} v_{\mu}$ decay and section 5 includes the study of LFU using $B^{0} \rightarrow D^{*-} \ell^{+} v_{\ell}$ and $B_{c}^{+} \rightarrow J / \psi \ell^{+} v_{\ell}$ decays. Finally, the conclusions are presented in section 6.

\section{Measurement of charmed baryons lifetimes}

The study of the lifetimes of heavy hadrons containing a $b$ or a $c$ quark allows to test the Heavy Quark Expansion (HQE), which can be used to calculate the decay widths of hadrons containing heavy quarks. While charmed-meson lifetimes have been measured precisely, the knowledge of charmed-baryon lifetimes is significantly worse. The lifetimes of the $D^{0}, D^{+}$and $D_{s}^{+}$mesons are known with $\sim 1 \%$ precision, whereas the uncertainties for the $\Lambda_{c}^{+}, \Xi_{c}^{+}, \Xi_{c}^{0}$ and $\Omega_{c}^{0}$ baryons are $3 \%$, $6 \%, 10 \%$ and $17 \%$, respectively [1]. It has been argued that the expected hierarchy for the charmed baryons lifetimes should be

$$
\tau_{\Xi_{c}^{+}}>\tau_{\Lambda_{c}^{+}}>\tau_{\Xi_{c}^{0}}>\tau_{\Omega_{c}^{0}}
$$

in agreement with the current measurements [1].

LHCb has recently measured the lifetimes of charmed baryons using inclusive semileptonic $H_{b} \rightarrow H_{c} \mu^{-} \bar{v}_{\mu}$ decays [2][3], where $H_{b}$ represents a $\Omega_{b}^{-}, \Lambda_{b}^{0}, \Xi_{b}^{0}$ or $\Xi_{b}^{-}$baryon and $H_{c}$ corresponds to a $\Omega_{c}^{0}, \Lambda_{c}^{+}, \Xi_{c}^{+}$or $\Xi_{c}^{0}$ baryon, respectively. The charmed baryons are reconstructed as $\Omega_{c}^{0} \rightarrow$ $p K^{-} K^{-} \pi^{+}, \Lambda_{c}^{+} \rightarrow p K^{-} \pi^{+}, \Xi_{c}^{+} \rightarrow p K^{-} \pi^{+}$and $\Xi_{c}^{0} \rightarrow p K^{-} K^{-} \pi^{+}$. To reduce the uncertainties associated with systematic effects, the lifetime ratios

$$
r_{H_{c}} \equiv \tau_{H_{c}} / \tau_{D^{+}}
$$

are measured, where the $D^{+}$meson is detected in $B \rightarrow D^{+} \mu^{-} \bar{v}_{\mu} X$ decays, with $D^{+} \rightarrow K^{-} \pi^{+} \pi^{+}$.

Proton-proton collision data collected by LHCb corresponding to $3 \mathrm{fb}^{-1}\left(1 \mathrm{fb}^{-1}\right.$ at $7 \mathrm{TeV}$ and $2 \mathrm{fb}^{-1}$ at $8 \mathrm{TeV}$ ) are used to reconstruct semileptonic decays including a hadron $H_{c}$ and a muon. The corresponding invariant masses are shown in Fig. 1. A binned maximum-likelihood fit is performed to each sample to obtain the signal yields. The $\Xi_{c}^{+}$and $\Xi_{c}^{0}$ yields are about 100 times larger than any previous sample used to measure the lifetimes of these baryons, the $\Lambda_{c}^{+}$sample is about 40 times larger and the number of $\Omega_{c}^{0}$ signal decays is at least one order of magnitude larger [2][3].

The decay-time spectra for the $H_{c}$ and $D^{+}$signals are shown in Fig. 2. The $H_{c}$ lifetimes are determined from simultaneous fits to the $H_{c}$ and $D^{+}$decay-time spectra, for which the free parameters in the fit are $r_{H_{c}}$ and $\tau_{D^{+}}$. 

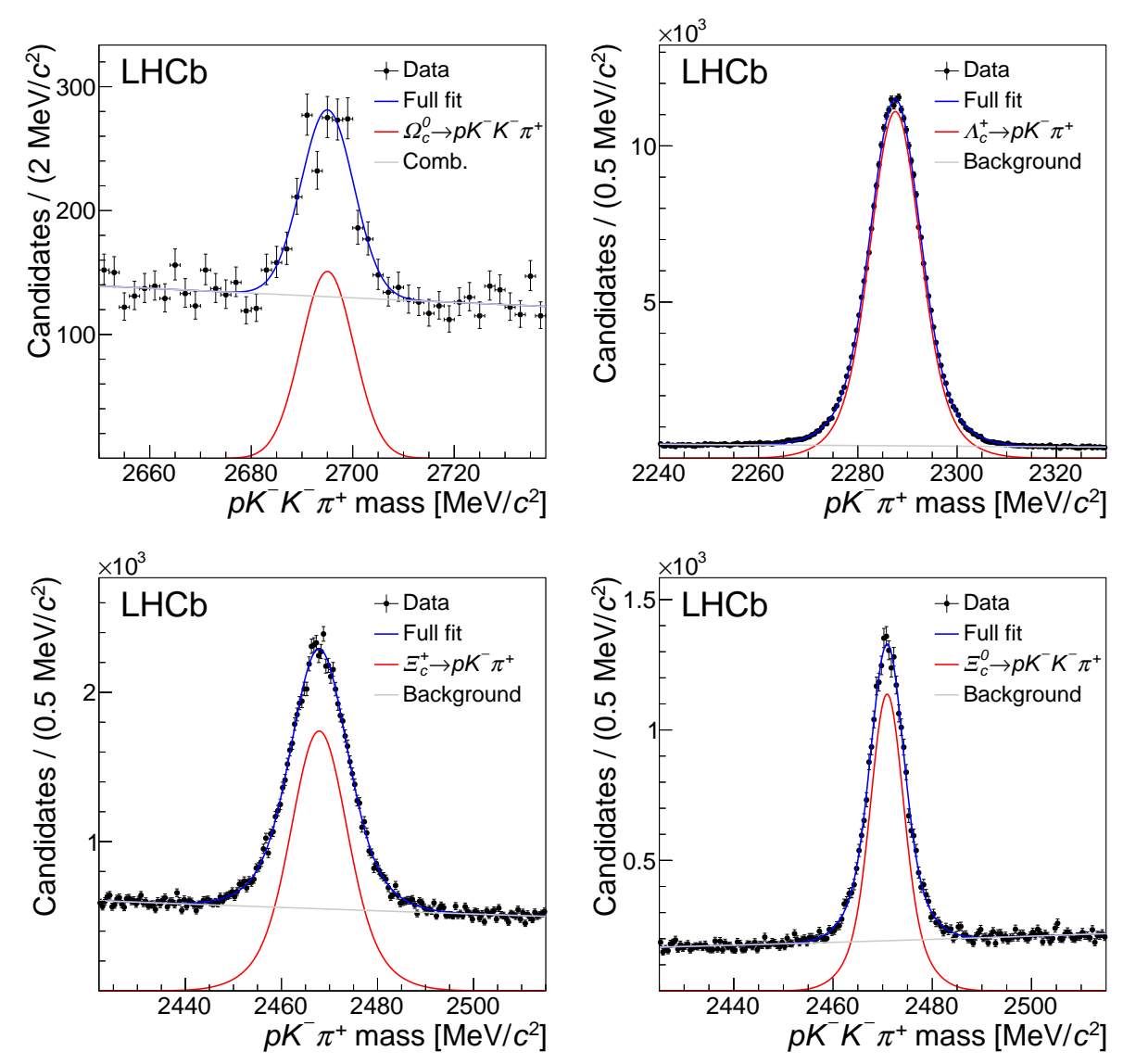

Figure 1: Invariant-mass distributions for (top left) $\Omega_{c}^{0}$, (top right) $\Lambda_{c}^{+}$, (bottom left) $\Xi_{c}^{+}$and (bottom right) $\Xi_{c}^{0}$ candidate decays. The results of the fits are overlaid.

The results of the fit to the $H_{c}$ decay-time distributions are shown in Fig. 2, where

$$
\begin{aligned}
& \tau_{\Omega_{c}^{0}}=268 \pm 24 \pm 10 \pm 2 \mathrm{fs} \\
& \tau_{\Lambda_{c}^{+}}=203.5 \pm 3.5 \pm 1.3 \pm 1.4 \mathrm{fs} \\
& \tau_{\Xi_{c}^{+}}=456.8 \pm 3.5 \pm 2.9 \pm 3.1 \mathrm{fs} \\
& \tau_{\Xi_{c}^{0}}=154.5 \pm 1.7 \pm 1.6 \pm 1.0 \mathrm{fs}
\end{aligned}
$$

are obtained. Here, the first uncertainty is statistical, the second systematic and the third due to the knowledge of the $D^{+}$lifetime.

The comparison between the measured lifetimes and the world averages is shown in Fig. 3. The $\Lambda_{c}^{+}$and $\Xi_{c}^{+}$lifetimes are measured with about $1 \%$ precision and are consistent with the existing world averages. The $\Xi_{c}^{0}$ lifetime is measured with about $1.8 \%$ precision, and is $3.3 \sigma$ larger than the world average value of $112_{-10}^{+13}$ fs. The measured $\Omega_{c}^{0}$ lifetime is about four times larger than, and inconsistent with, the world average value of $69 \pm 12$ fs [1]. These results are in disagreement with the expected hierarchy (Eq. (2.1)) and may suggest that additional or higher order contributions in HQE need to be considered. 

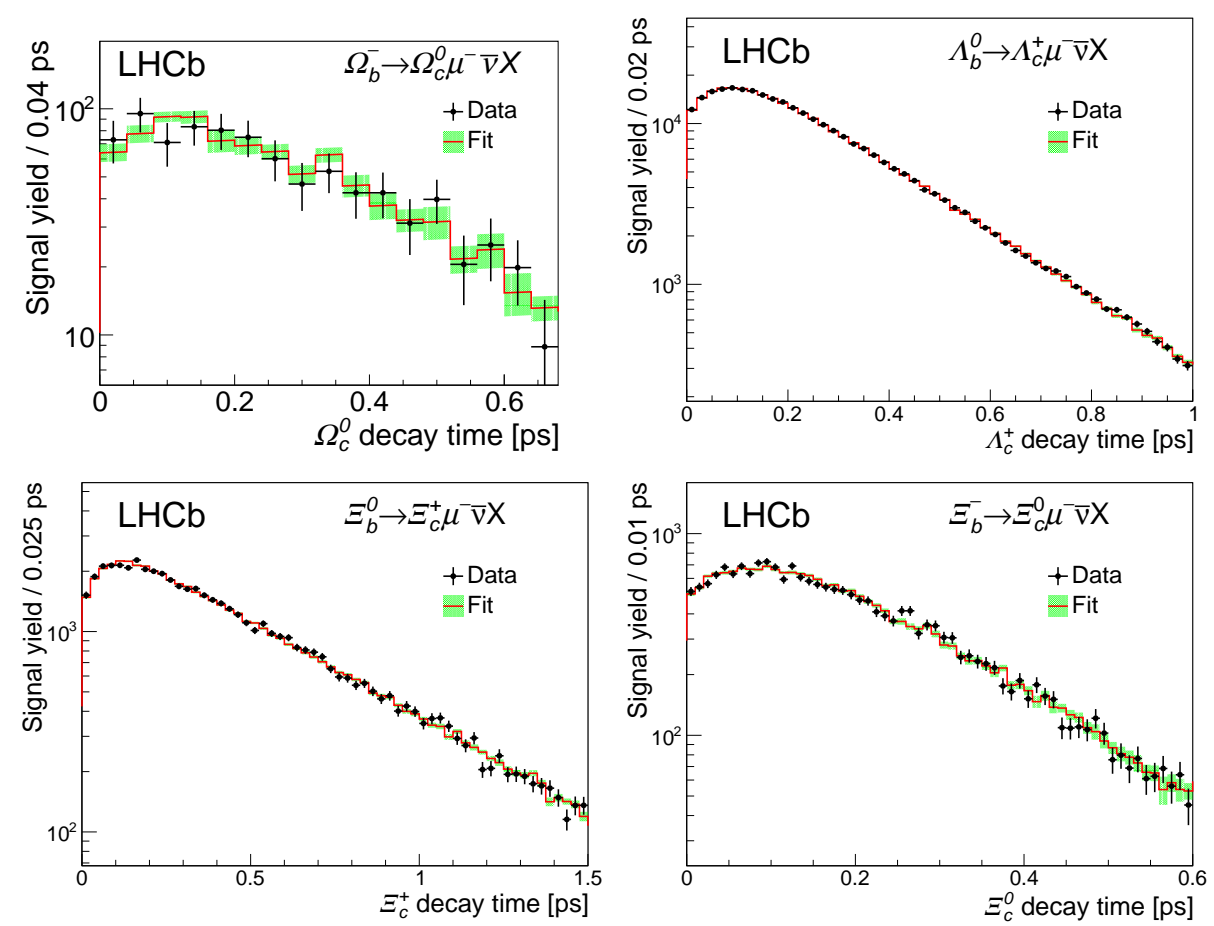

Figure 2: Decay-time distributions for candidate (top left) $\Omega_{c}^{0}$, (top right) $\Lambda_{c}^{+}$, (bottom left) $\Xi_{c}^{+}$and (bottom right) $\Xi_{c}^{0}$ candidate decays. The results of the fits are overlaid.

\section{Determination of $\left|V_{u b}\right|$ using $\Lambda_{b}^{0} \rightarrow p \mu^{-} \bar{v}_{\mu}$ decays}

The study of semileptonic $b \rightarrow u \ell^{-} \bar{v}_{\ell}$ transition allows for a determination of the CKM matrix element $\left|V_{u b}\right|$. Using $3 \mathrm{fb}^{-1}$ of integrated luminosity collected by LHCb in proton-proton collisions, the ratio of branching fractions $\Lambda_{b}^{0} \rightarrow p \mu^{-} \bar{v}_{\mu}$ and $\Lambda_{b}^{0} \rightarrow \Lambda_{c}^{+} \mu^{-} \bar{v}_{\mu}$ is measured [4] to obtain the ratio

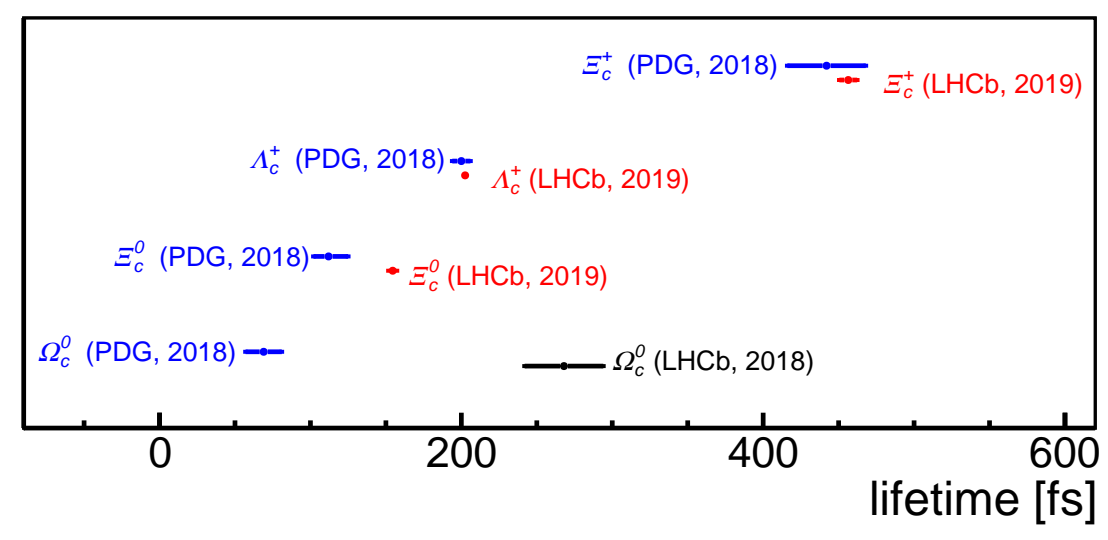

Figure 3: Comparison between the world average (blue) for charmed baryons lifetimes and the measured lifetimes (red and black). 
$\left|V_{u b}\right|^{2} /\left|V_{c b}\right|^{2}$ according to

$$
\frac{\left|V_{u b}\right|^{2}}{\left|V_{c b}\right|^{2}}=\frac{\mathscr{B}\left(\Lambda_{b}^{0} \rightarrow p \mu^{-} \bar{v}_{\mu}\right)}{\mathscr{B}\left(\Lambda_{b}^{0} \rightarrow \Lambda_{c}^{+} \mu^{-} \bar{v}_{\mu}\right)} R_{F F}
$$

where $\mathscr{B}$ denotes the branching fraction and $R_{F F}$ is a ratio of form factors, calculated using Lattice QCD (LQCD). This is then converted into a measurement of $\left|V_{u b}\right|$ using the existing measurements of $\left|V_{c b}\right|$ from exclusive decays.

Signal $\Lambda_{b}^{0} \rightarrow p \mu^{-} \bar{v}_{\mu}$ candidates are reconstructed by requiring two tracks compatible with being a proton and a muon forming a good $p \mu^{-}$vertex. For the normalisation, $\Lambda_{b}^{0} \rightarrow \Lambda_{c}^{+} \mu^{-} \bar{v}_{\mu}$ candidates are formed adding two tracks, identified as a pion and a kaon to fully reconstruct the $\Lambda_{c}^{+} \rightarrow p K^{-} \pi^{+}$decay. The $\Lambda_{b}^{0}$ corrected mass is used to determine the signal and normalisation yields. This is defined as

$$
M_{\text {corr }}=\sqrt{m_{h \mu}^{2}+p_{\perp}^{2}}+p_{\perp}
$$

where $m_{h \mu}$ is the mass of the $h \mu$ pair and $p_{\perp}$ is the momentum of the $h \mu$ pair transverse to the $\Lambda_{b}^{0}$ direction of flight, where $h$ represents either the proton or the $\Lambda_{c}^{+}$candidate.

The squared of the invariant mass of the $\mu^{-} \bar{v}_{\mu}$ pair, $q^{2}$, is determined using the $\Lambda_{b}^{0}$ direction of flight and the $\Lambda_{b}^{0}$ nominal mass up to a two-fold ambiguity. Both solutions are required to exceed 15 (7) $\mathrm{GeV}^{2} / c^{4}$ for the $\Lambda_{b}^{0} \rightarrow p \mu^{-} \bar{v}_{\mu}\left(\Lambda_{b}^{0} \rightarrow \Lambda_{c}^{+} \mu^{-} \bar{v}_{\mu}\right)$ candidates. These are the regions where the corresponding form-factors can be calculated more precisely.

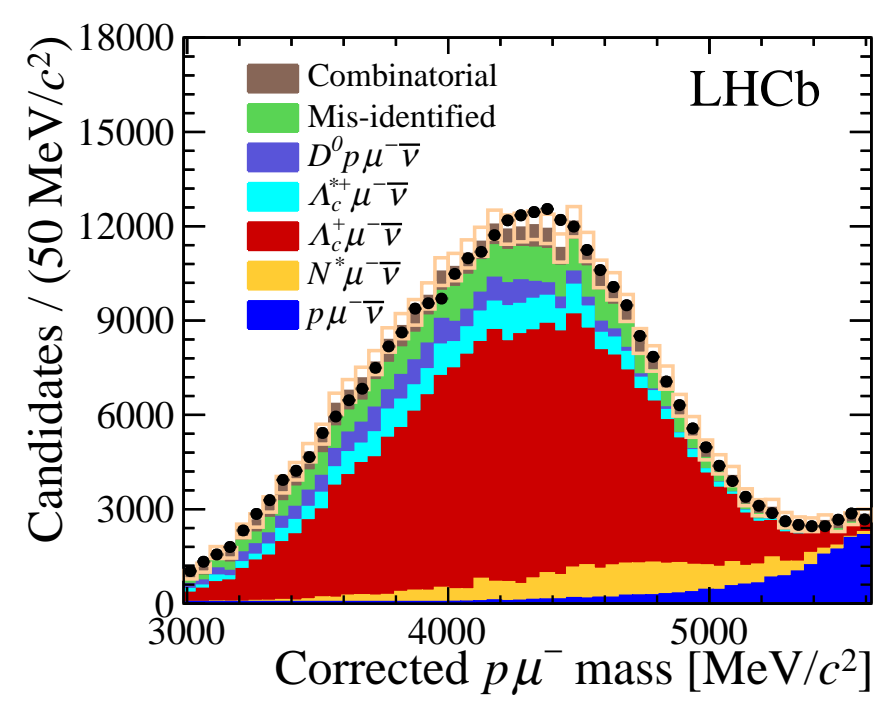

Figure 4: Corrected mass fit to $\Lambda_{b}^{0} \rightarrow p \mu^{-} \bar{v}_{\mu}$ candidates. The fit components are shown as labels overlaid in the figure. Signal $\Lambda_{b}^{0} \rightarrow p \mu^{-} \bar{v}_{\mu}$ is clearly seen as a blue component at high values of the corrected mass.

The mass distribution of the signal candidates is shown in Fig. 4. The signal and normalisation yields are determined from separate $\chi^{2}$ fits to the $M_{\text {corr }}$ distributions of the $\Lambda_{b}^{0} \rightarrow p \mu^{-} \bar{v}_{\mu}$ and $\Lambda_{b}^{0} \rightarrow \Lambda_{c}^{+}\left(\rightarrow p K^{-} \pi^{+}\right) \mu^{-} \bar{v}_{\mu}$ candidates. The shapes of the signal, normalisation and backgrounds are modelled using simulation. The $\Lambda_{b}^{0} \rightarrow p \mu^{-} \bar{v}_{\mu}$ branching fraction is measured relative to the 
$\Lambda_{b}^{0} \rightarrow \Lambda_{c}^{+}\left(\rightarrow p K^{-} \pi^{+}\right) \mu^{-} \bar{v}_{\mu}$ branching fraction, using the determined yields and corrected by the relative efficiencies. The result

$$
\frac{\mathscr{B}\left(\Lambda_{b}^{0} \rightarrow p \mu^{-} \bar{v}_{\mu}\right)_{q^{2}>15}}{\mathscr{B}\left(\Lambda_{b}^{0} \rightarrow \Lambda_{c}^{+} \mu^{-} \bar{v}_{\mu}\right)_{q^{2}>7}}=(1.00 \pm 0.04 \pm 0.08) \times 10^{-2}
$$

is obtained. Using Eq. (3.1) with $R_{F F}=0.68 \pm 0.07$, computed in Ref. [5], the measurement

$$
\frac{\left|V_{u b}\right|}{\left|V_{c b}\right|}=0.083 \pm 0.004 \pm 0.004
$$

is obtained. The first uncertainty arises from the experimental measurement and the second is due to the uncertainty in the form-factors prediction. Finally, using the world average $\left|V_{c b}\right|=$ $(39.5 \pm 0.8) \times 10^{-3}$ measured using exclusive decays, $\left|V_{u b}\right|$ is measured as

$$
\left|V_{u b}\right|=(3.27 \pm 0.15 \pm 0.16 \pm 0.06) \times 10^{-3},
$$

where the first uncertainty is due to the experimental measurement, the second arises from the uncertainty in the LQCD prediction and the third from the normalisation to $\left|V_{c b}\right|$. This measurement is in agreement with the world average using exclusive decays and in tension with the measurement using inclusive decays [1].

\section{Search for the decay $B^{-} \rightarrow \mu^{+} \mu^{-} \mu^{-} \bar{v}_{\mu}$}

Decays of $B^{+} \rightarrow \tau^{+} v_{\tau}$ and $B^{+} \rightarrow \mu^{+} v_{\mu}$ are CKM and helicity suppressed, which make them sensitive to new particles such as charged scalars. In addition, they have precise SM predictions due to the absence of hadrons in the final state.

The decay $B^{+} \rightarrow \mu^{+} v_{\mu} \gamma$ is important because it is a background for the $B^{+} \rightarrow \mu^{+} v_{\mu}$ decay and its branching fraction is a direct measurement of the inverse moment of the $B$ meson light cone distribution amplitude. However, a vertex with only a single charged particle is impossible in the LHC environment. This problem is not present in the $B^{+} \rightarrow \mu^{+} \mu^{-} \mu^{+} v_{\mu}$ decay, which receives contributions from the $B^{+} \rightarrow \mu^{+} v_{\mu} \gamma^{*}\left(\rightarrow \mu^{+} \mu^{-}\right)$and $B^{+} \rightarrow \mu^{+} v_{\mu} V$ amplitudes, where $V$ is a vector meson ( $\omega$ or $\rho$ ) decaying to two muons.

A search for the $B^{+} \rightarrow \mu^{+} \mu^{-} \mu^{+} v_{\mu}$ decay has been performed at LHCb [6] using $4.7 \mathrm{fb}^{-1}$ of integrated luminosity. The selection requires a good quality vertex formed by three muons, well separated from the primary vertex. The candidate is required to be in the region below $980 \mathrm{MeV} / \mathrm{c}^{2}$ for the lower of the two $\mu^{+} \mu^{-}$mass combinations. The distribution of the corrected mass

$$
M_{\text {corr }}=\sqrt{M_{\mu \mu \mu}^{2}+p_{\perp}^{2}}+p_{\perp},
$$

is used to peform a fit to obtain the signal yield. Here, $p_{\perp}$ is the component of the momentum perpendicular to the $B^{+}$direction of flight and $M_{\mu \mu \mu}$ is the invariant mass of the three muons.

The result of the fit is shown in Fig. 5. The determined signal yield is $-25 \pm 16$, resulting in a limit on the branching fraction of

$$
\mathscr{B}\left(B^{+} \rightarrow \mu^{+} \mu^{-} \mu^{+} v_{\mu}\right)<1.6 \times 10^{-8}
$$

at $95 \%$ confidence level. This limit is in tension with a theoretical calculation based on the vectordominance model [7]. 


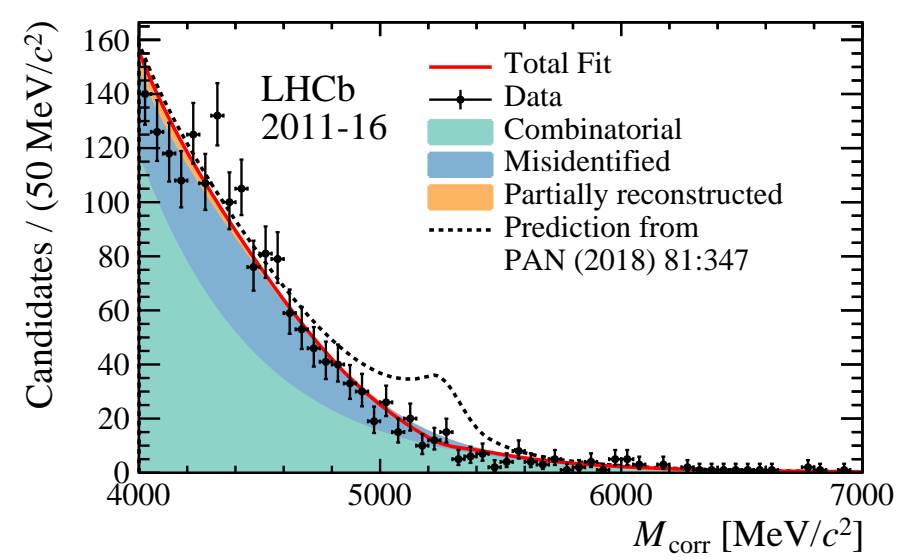

Figure 5: Corrected mass distribution of $B^{+} \rightarrow \mu^{+} \mu^{-} \mu^{+} v_{\mu}$ candidates. The overlaid fit (red line) is composed of (green) combinatorial background, (blue) misidentified candidates and (orange) partically reconstructed events. The signal component is not visible as the fitted signal yield is negative. The dashed line shows how the total model would look like if the signal had the branching fraction predicted in Ref. [7].

\section{Lepton Flavour Universality tests using semitauonic $B$ decays}

In the SM, couplings between the gauge bosons and the three families of leptons are equal. This feature is known as lepton flavour universality. Some models beyond the SM predict that new physics is coupled preferentially to the third generation lepton, the $\tau$. Semitauonic decays of $b$ hadrons provide a sensitive probe for such effects.

LHCb has performed three studies of LFU using semitauonic $b$-hadron decays:

- Study of $R\left(D^{*}\right)=\mathscr{B}\left(B^{0} \rightarrow D^{*-} \tau^{+} v_{\tau}\right) / \mathscr{B}\left(B^{0} \rightarrow D^{*-} \mu^{+} v_{\mu}\right)$ using leptonic $\tau^{-} \rightarrow \mu^{-} \bar{v}_{\mu} v_{\tau}$ decays [8].

- Study of $R\left(D^{*}\right)$ using hadronic $\tau^{-} \rightarrow \pi^{+} \pi^{-} \pi^{+}\left(\pi^{0}\right) v_{\tau}$ decays [9][10].

- Study of $R(J / \psi)=\mathscr{B}\left(B_{c}^{+} \rightarrow J / \psi \tau^{+} v_{\tau}\right) / \mathscr{B}\left(B_{c}^{+} \rightarrow J / \psi \mu^{+} v_{\mu}\right)$ using leptonic $\tau^{-} \rightarrow \mu^{-} \bar{v}_{\mu} v_{\tau}$ decays [11].

The study of $B_{c}^{+} \rightarrow J / \psi \tau^{+} v_{\tau}$ decays resulted in the first evidence for this decay and a value for $R(J / \psi)$ of $0.71 \pm 0.17$ (stat) \pm 0.18 (syst), about $1.7 \sigma$ above the range of central values currently predicted in the SM. The study of $R\left(D^{*}\right)$ using muonic $\tau$ decays results in $R\left(D^{*}\right)=0.336 \pm$ 0.027 (stat) \pm 0.030 (syst), $2.1 \sigma$ above the SM. The corresponding analysis using 3-prong hadronic $\tau$ decays provides $R\left(D^{*}\right)=0.291 \pm 0.019$ (stat) \pm 0.026 (syst) \pm 0.013 (ext), compatible with the SM at $1 \sigma$ level. The LHCb $R\left(D^{*}\right)$ measurements together with the measurements of $R\left(D^{*}\right)$ and $R(D)$ performed at the B-factories are shown in Fig. 6. Currently, the tension between the world average and the SM prediction is about 3.1 $\sigma$. Finally, figure 7 shows the expected evolution of the uncertainty for $R\left(D^{*}\right)$ and $R(J / \psi)$ at LHCb with respect to the integrated luminosity to be collected in the LHCb upgrades [12]. 


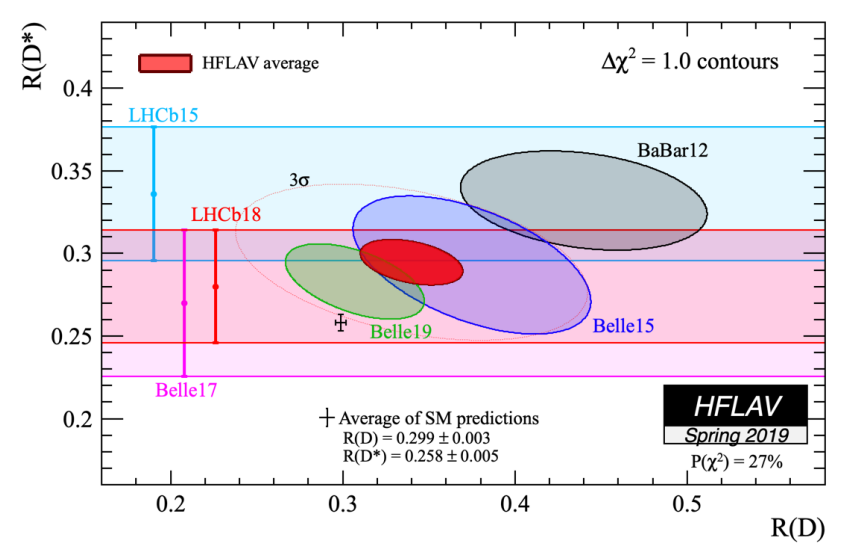

Figure 6: Measurements of $R\left(D^{*}\right)$ and $R(D)$ peformed by $\mathrm{LHCb}$ and the $\mathrm{B}$-factories. The world average is shown as a red ellipse and the SM prediction as a black cross. Figure taken from HFLAV [13] (https://hflaveos.web.cern.ch/hflav-eos/semi/spring 19/main.shtml).

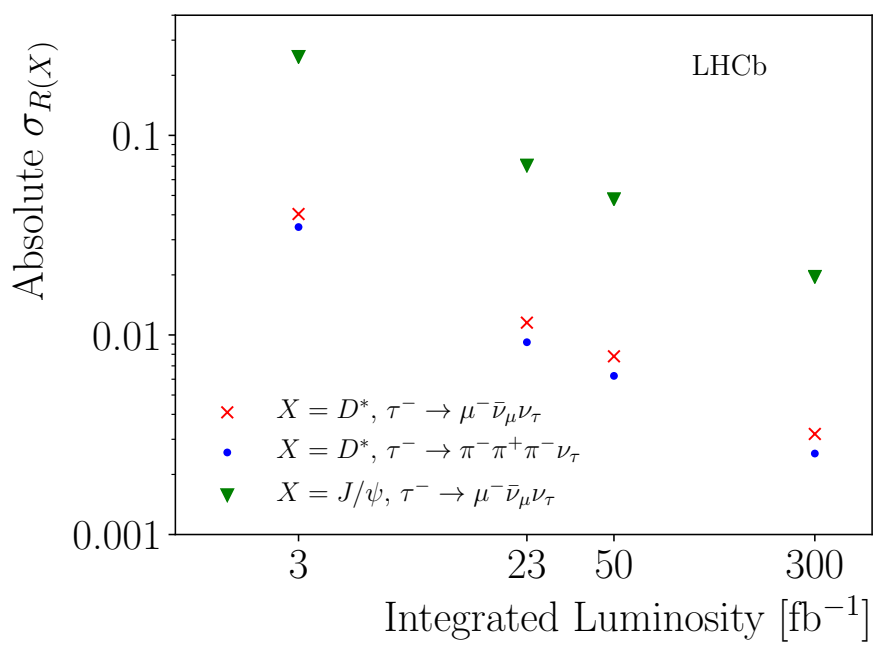

Figure 7: Evolution of the uncertainty in $R\left(D^{*}\right)$ and $R(J / \psi)$ as a function of the integrated luminosity expected to be collected by LHCb.

\section{Conclusions}

Measurements performed by the LHCb experiment in semileptonic $b$-hadron decays are presented. These include precise measurements of charmed baryons lifetimes, the measurement of $\left|V_{u b}\right|$ using semileptonic $\Lambda_{b}^{0}$ decays and tests of LFU using semitauonic $b$-hadron decays. The measurement of the $\Omega_{c}^{0}$ baryon lifetime is found to be incompatible with the current world average, and the $\Xi_{c}^{0}$ shows a $3.3 \sigma$ deviation. On the other hand, the measurements of $R\left(D^{*}\right)$ and $R(J / \psi)$ were performed using $3 \mathrm{fb}^{-1}$ of integrated luminosity. The inclusion of the full LHCb dataset $\left(9 \mathrm{fb}^{-1}\right)$ and the data to be collected after the LHCb upgrades ( $300 \mathrm{fb}^{-1}$ at the end of Upgrade-II) will allow to measure these observables with few percent precision. 


\section{References}

[1] Particle Data Group, M. Tanabashi et al. Review of particle physics, Phys. Rev. D98 (2018) 030001.

[2] R. Aaij et al., LHCb Collaboration. Precision measurement of the $\Lambda_{c}^{+}, \Xi_{c}^{+}$and $\Xi_{c}^{0}$ baryon lifetimes, Phys.Rev. D100 (2019) 032001, arXiv:1906.08350.

[3] R. Aaij et al., LHCb Collaboration. Measurement of the $\Omega_{c}^{0}$ baryon lifetime, Phys.Rev.Lett. 121 (2018) no.9, 092003, arXiv:1807.02024.

[4] R. Aaij et al., LHCb Collaboration. Determination of the quark coupling strength $\left|V_{u b}\right|$ using baryonic decays, Nature Phys. 11 (2015) 743-747, arXiv:1504.01568.

[5] Detmold, William and Lehner, Christoph and Meinel, Stefan. $\Lambda_{b} \rightarrow p \ell^{-} \bar{v}_{\ell}$ and $\Lambda_{b} \rightarrow \Lambda_{c} \ell^{-} \bar{v}_{\ell}$ form factors from lattice QCD with relativistic heavy quarks, Phys.Rev. D92 (2015) no.3, 034503, arXiv:1503.01421.

[6] R. Aaij et al., LHCb Collaboration. Search for the rare decay $B^{+} \rightarrow \mu^{+} \mu^{-} \mu^{+} v_{\mu}$, arXiv:1812.06004.

[7] Danilina, A. V. and Nikitin, N. V. Four-Leptonic Decays of Charged and Neutral B Mesons within the Standard Model, Phys.Atom.Nucl. 81 (2018) no.3, 347-359 [Yad.Fiz. 81 (2018) no.3, 331-345].

[8] R. Aaij et al., LHCb Collaboration. Measurement of the ratio of branching fractions $\mathscr{B}\left(\bar{B}^{0} \rightarrow D^{*+} \tau^{-} \bar{v}_{\tau}\right) / \mathscr{B}\left(\bar{B}^{0} \rightarrow D^{*+} \mu^{-} \bar{v}_{\mu}\right)$, Phys.Rev.Lett. 115 (2015) no.11, 111803, arXiv:1506.08614.

[9] R. Aaij et al., LHCb Collaboration. Measurement of the ratio of the $B^{0} \rightarrow D^{*-} \tau^{+} v_{\tau}$ and $B^{0} \rightarrow D^{*-} \mu^{+} v_{\mu}$ branching fractions using three-prong $\tau$-lepton decays, Phys.Rev.Lett. 120 (2018) no.17, 171802, arXiv:1708.08856.

[10] R. Aaij et al., LHCb Collaboration. Test of Lepton Flavor Universality by the measurement of the $B^{0} \rightarrow D^{*-} \tau^{+} v_{\tau}$ branching fraction using three-prong $\tau$ decays, Phys.Rev. D97 (2018) no.7, 072013, arXiv:1711.02505.

[11] R. Aaij et al., LHCb Collaboration. Measurement of the ratio of branching fractions $\mathscr{B}\left(B_{c}^{+} \rightarrow J / \psi \tau^{+} v_{\tau}\right) / \mathscr{B}\left(B_{c}^{+} \rightarrow J / \psi \mu^{+} v_{\mu}\right)$, Phys.Rev.Lett. 120 (2018) no.12, 121801, arXiv:1711.05623.

[12] R. Aaij et al., LHCb Collaboration. Physics case for an LHCb Upgrade II - Opportunities in flavour physics, and beyond, in the HL-LHC era, arXiv:1808.08865.

[13] Amhis, Y. and others, Heavy Flavor Averaging Group. Averages of b-hadron, c-hadron, and $\tau$-lepton properties as of summer 2016, Eur. Phys. J. C (2017) 77: 895, arXiv:1612.07233. Online updates in https://hflav.web.cern.ch/. 